\title{
Neurobiology of suicide: do biomarkers exist?
}

\author{
Alessandra Costanza • Isabella D'Orta • Nader Perroud • \\ Sandra Burkhardt • Alain Malafosse • Patrice Mangin • \\ Romano La Harpe
}

Received: 28 November 2012 / Accepted: 5 February 2013 /Published online: 22 February 2013

(C) Springer-Verlag Berlin Heidelberg 2013

\begin{abstract}
Clinical risk factors have a low predictive value on suicide. This may explain the increasing interest in potential neurobiological correlates and specific heritable markers of suicide vulnerability. This review aims to present the current neurobiological findings that have been shown to be implicated in suicide completers and to discuss how postmortem studies may be useful in characterizing these individuals. Data on the role of the main neurobiological systems in suicidality, such as the neurotransmitter families, hypothalamic-pituitary-adrenal axis, neurotrophic factors, and polyamines, are exposed at the different biochemical, genetic, and epigenetic levels. Some neuroanatomic and neuropathological aspects as well as their in vivo morphological and functional neuroimaging correlates are also described. Except for the serotoninergic system, particularly with respect to the polymorphism of the gene coding for the serotonin transporter (5-HTTLPR) and brain-derived neurotrophic factor, data did not converge to
\end{abstract}

Electronic supplementary material The online version of this article (doi:10.1007/s00414-013-0835-6) contains supplementary material, which is available to authorized users.

A. Costanza $\cdot$ I. D’Orta $\cdot$ N. Perroud

Department of Mental Health and Psychiatry,

University Hospitals of Geneva, Geneva, Switzerland

N. Perroud $(\bowtie) \cdot$ A. Malafosse

Department of Psychiatry, University of Geneva,

Hôpital de Belle-Idée, 2 ch. du Petit-Bel-Air,

1225 Chêne-Bourg, Switzerland

e-mail: nader.perroud@hcuge.ch

A. Malafosse

Department of Medical Genetic and Laboratories,

University Hospitals of Geneva, Geneva, Switzerland

S. Burkhardt $\cdot$ P. Mangin $\cdot$ R. La Harpe

University Center of Legal Medicine, Lausanne-Geneva (CMU),

9, av. de Champel,

CH-1211 Geneva 4, Switzerland produce a univocal consensus. The possible limitations of currently published studies are discussed, as well as the scope for long-term prospective studies.

Keywords Suicide · Suicide behavior · Suicide completion · Biomarkers

\section{Introduction}

Worldwide, more people die by suicide every year than by homicides and in all wars combined [S1]. The estimated global burden of suicide is a million deaths per year, representing a mortality rate of 14.5 deaths per 100,000 habitants per year [1, S2].

Suicide has been variously defined in the literature and the standardization of its nomenclature is being debated [S3], notably for aspects referring to intentionality [S4]. A widely utilized definition describes suicide as a "fatal selfinflicted self-destructive act with explicit or inferred intent to die" [S5]. In forensic medicine, to distinguish suicide from deaths due to other circumstances, three main components are considered: (1) death as the result of injuries, poisoning, or suffocation; (2) self-inflicted; and (3) intentionally inflicted [S3]. These operational criteria, however, present some limitations, and considerable variability has been observed in the way in which medicolegal experts define a person who committed suicide [S6-S9].

Suicide (also called suicide completion) represents a complex phenomenon, placed at the extreme of a continuum of behaviors commonly referred to as suicidal behaviors (SB) which also includes suicide attempt and suicidal ideas [2, S4]. A suicide attempt, or nonfatal $\mathrm{SB}$, is "a potentially selfinjurious behavior with a nonfatal outcome, for which there is evidence (either explicit or implicit) that the person intended at some level to kill himself/herself; a suicide attempt may or 
may not result in injuries" [S3]. However, suicide attempters and suicide completers only share a part of the underlying etiological and neurobiological mechanisms and suicide completers are considered a more homogeneous group than suicide attempters [2].

Risk factors for suicide can be categorized as distal and proximal factors [2,3]. Distal risk factors include family history of suicide and genetic loading, early life adversities and epigenetic modifications, personality traits, and cognitive styles $[2$, 3]. Family history of suicide is thought to be partially independent from familial clustering of mental disorders [S10, S11]. The presence of early traumatic life events is considered as one of the strongest distal risk factors for both suicide attempts and suicide completion in adulthood [4, S12-S14]. Proximal risk factors are represented by conditions that act as precipitants: existence of psychiatric and/or physical disorders, psychosocial crisis, recent life events conferring acute stress, and availability of means $[2,3]$. A number of sociodemographic factors can be considered to be moderators of the relationship between distal and proximal risk factors [2], such as gender, age, education, religious and spiritual beliefs, family structure, employment and income, social support, and quality of social environment [5, S15-S18]. The relation between neurobiological risk factors and stressors has been described in some explanatory models [S19], such as the stress-diathesis model [6, S20, S21]. In this model, hopelessness, impulsivity, and aggression are components of the diathesis for SB.

Despite the existence of these numerous clinical risk factors, none actually help to define who will die by suicide or not. The low predictive value of clinical risk factors for suicide completions may explain the increasing interest in investigating potential neurobiological correlates and specific heritable markers of suicide vulnerability. Association mapping and family, twin, and adoption studies strongly suggest heritability to suicide. Genetic studies conducted to date have mainly focused on the serotonin (5-HT) system and identified some related candidate genes [S4, S22]. Several other genes have been highlighted either through genome-wide association or microarray studies with more or less satisfactory results [7-10, S23-S28]. Finally, the search for biological correlates of suicide has more recently been expanded by epigenetic as well as exome sequencing and microRNA studies [7-10, S23-S29] (see Glossary [S30]).

The aims of the present review are to present the main current neurobiological aspects that have been shown to be implicated in suicide completion and to discuss how postmortem studies may be useful in characterizing these individuals and how they may contribute to helping the medical expert to determine the circumstances of death, namely, if suicide is suspected. In this respect, findings on the role of the neurotransmitter systems, hypothalamic-pituitary-adrenal (HPA) axis, neurotrophic factors, and polyamines will be discussed at the different biochemical, genetic, and epigenetic levels
(Table 1). A final section will be dedicated to neuroanatomic and neuropathological data in suicide completers (Fig. 1) as well their correlates to in vivo structural and functional imaging results.

\section{Neurotransmitter systems}

\section{Serotonin}

5-HT is assumed to play a major role in the neurobiological basis of SB. Indeed, 5-HT system alterations are known to be part of the neurobiological diathesis of suicide mentioned previously [6]. Abnormalities in this system have been found in the prefrontal cortex (PFC), hypothalamus, and brainstem of suicide victims (independently of a history of mood disorder), especially in the ventral prefrontal cortex (VPFC) [11, S31-S33].

Low levels of 5-hydroxyindoleacetic acid (5-HIAA), the main 5-HT metabolite, in cerebrospinal fluid (CSF) have been found in persons who had made a violent suicide attempt [S34]. It has been suggested that low CSF 5-HIAA is specific to suicide as it was found only in suicidal patients [12]. It has been postulated that this biochemical trait may be considered a predictor of suicide attempts and completions; it is consistent with low postmortem brainstem levels of 5-HIAA in suicide victims [6]. It has also been shown that patients with a history of high-lethality suicide attempts had a blunted response to fenfluramine, an indicator of central 5-HT activity through prolactin secretion, independently of the comorbid psychiatric disorders [S35].

These findings have induced molecular genetic studies to focus on 5-HT-related genes in search of genetic markers of suicide. Tryptophan hydroxylase 1 (TPH1), tryptophan hydroxylase 2 (TPH2), and 5-HT transporter (5-HTT; SLC6A4) genes have received the most research attention [13, S36, S37]. Two different isoforms of TPH, the rate-limiting enzyme in the biosynthesis of 5-HT, are located on two different chromosomes. TPH1 is located on chromosome 11 and is responsible for peripheral 5-HT generation, while $T P H 2$ is known as the brain-specific isoform and is located on chromosome 12 [S38]. Initially, studies of postmortem brains of suicides found no evidence to support the involvement of TPH in suicide: no difference in TPH immunoreactivity emerged between suicide victims and controls, even when investigating specific subnuclei of the dorsal raphe [S39]. More recently, in contrast, TPH immunoreactivity was found to be higher in the dorsal raphe nucleus of suicide victims, as a possible compensatory mechanism to balance the diminished 5-HT activity [S40]. Higher levels of THP2 mRNA were found in the dorsal and raphe nucleus of drug-free suicide subjects [S41]. Several polymorphisms in THP1 have been associated with suicide attempt, but to our knowledge, none have truly been implicated in 
Table 1 Overview of the main findings in suicide completers at the biochemical, genetic, and epigenetic levels

\begin{tabular}{|c|c|}
\hline & Authors \\
\hline \multicolumn{2}{|l|}{ Proteins } \\
\hline 5-HIAA & {$[6,12, \mathrm{~S} 34]$} \\
\hline 5-HTT & {$[11,12, \mathrm{~S} 32, \mathrm{~S} 34]$} \\
\hline 5-HTR-1A, 5-HTR-2A & {$[\mathrm{S} 31, \mathrm{~S} 52, \mathrm{~S} 53, \mathrm{~S} 58]$} \\
\hline AMPA receptors & [S64] \\
\hline$\mu$-Opioids receptors & {$[\mathrm{S} 68, \mathrm{~S} 69]$} \\
\hline $\mathrm{CRH}$ & {$[\mathrm{S} 78]$} \\
\hline BDNF & {$[20,23, \mathrm{~S} 82, \mathrm{~S} 85]$} \\
\hline TrkB & {$[\mathrm{S} 82, \mathrm{~S} 89]$} \\
\hline NT-3 & {$[\mathrm{S} 85]$} \\
\hline PKA, PKC & [S91-S94] \\
\hline CREB & {$[\mathrm{S} 95, \mathrm{~S} 96, \mathrm{~S} 98]$} \\
\hline $\mathrm{PKB} / \mathrm{akt}$ & {$[21, \mathrm{~S} 99]$} \\
\hline GSK-3 $\beta$ & [S99] \\
\hline PI3K & {$[21]$} \\
\hline PTEN & {$[21]$} \\
\hline QKI & [S119] \\
\hline A2-adrenergic receptors & {$[11, \mathrm{~S} 65]$} \\
\hline \multicolumn{2}{|l|}{ mRNA } \\
\hline 5-HTT/SLC6A4 mRNA & {$[13]$} \\
\hline 5-HTR-2A mRNA, 5-HTR-2C mRNA & {$[\mathrm{S} 58]$} \\
\hline TPH1 mRNA,TPH2 mRNA & {$[13, \mathrm{~S} 38, \mathrm{~S} 41, \mathrm{~S} 43]$} \\
\hline CRH mRNA & {$[\mathrm{S} 74, \mathrm{~S} 75, \mathrm{~S} 78, \mathrm{~S} 79]$} \\
\hline CRH receptor mRNA & {$[\mathrm{S} 77, \mathrm{~S} 80]$} \\
\hline POMC mRNA & {$[\mathrm{S} 75]$} \\
\hline GR mRNA, GR $1_{F}$ mRNA & {$[18]$} \\
\hline $\mathrm{GR} 1_{\mathrm{B}}, 1_{\mathrm{C}}, 1_{\mathrm{H}} \mathrm{mRNA}$ & [19] \\
\hline BDNF mRNA & [S82] \\
\hline TrkB mRNA & [S82] \\
\hline PKA mRNA & {$[\mathrm{S} 93, \mathrm{~S} 94]$} \\
\hline CREB mRNA & {$[\mathrm{S} 96, \mathrm{~S} 98]$} \\
\hline P11 mRNA & {$[22]$} \\
\hline SSAT mRNA & {$[\mathrm{S} 27, \mathrm{~S} 104, \mathrm{~S} 105]$} \\
\hline QKI mRNA & {$[\mathrm{S} 119]$} \\
\hline microRNA & [S29] \\
\hline \multicolumn{2}{|l|}{ Candidate genes } \\
\hline 5-HTT/SLC6A4 (5-HTTLPR) & {$[11, \mathrm{~S} 50, \mathrm{~S} 51]$} \\
\hline 5-HTR-1A, 5-HTR-2A & {$[\mathrm{S} 24, \mathrm{~S} 52]$} \\
\hline TPH1,ТPH2 & {$[13, \mathrm{~S} 42, \mathrm{~S} 44]$} \\
\hline GABRA, GABA transporter & {$[8,9$, S25, S26, S62, S63] } \\
\hline GR & {$[18,19]$} \\
\hline BDNF & {$[S 82, \mathrm{~S} 84]$} \\
\hline TrkB, TrkB-T1 & {$[\mathrm{S} 82, \mathrm{~S} 84, \mathrm{~S} 87, \mathrm{~S} 88]$} \\
\hline FGFR3, FGFR2 & {$[7, \mathrm{~S} 63]$} \\
\hline CREB1 & [S97] \\
\hline SSAT & {$[S 27, \mathrm{~S} 104, \mathrm{~S} 105]$} \\
\hline QKI & {$[\mathrm{S} 119]$} \\
\hline
\end{tabular}

Table 1 (continued)

\begin{tabular}{ll}
\hline & Authors \\
\hline $\begin{array}{l}\text { Multiple genes expression profiling } \\
\text { studies }\end{array}$ & {$[8-10, \mathrm{~S} 23-\mathrm{S} 25, \mathrm{~S} 27$,} \\
Epigenetic changes & \\
GR, GR exon $1_{\mathrm{F}}$ & {$[18,19]$} \\
BDNF & {$[20]$} \\
TrkB-T1 & {$[\mathrm{S} 87, \mathrm{~S} 88]$} \\
SSAT & {$[24], \mathrm{S} 109$}
\end{tabular}

$A M P A$ receptor ionotropic receptor for glutamate, $B D N F$ brain-derived neurotrophic factor, $C R E B$ cyclic AMP-responsive element binding, $C R H$ corticotrophin-releasing hormone, FGFR fibroblast growth factor, GABRA GABA receptor, GR glucocorticoid receptor, GSK$3 \beta$ glycogen synthase kinase-3 $\beta, 5$-HIAA 5-hydroxyindoleacetic acid, 5 HTR-1A, 5-HTR-2A, and 5-HTR-2C serotonin receptors, 5-HTT serotonin transporter, 5-HTTLPR polymorphism of the HTT/SLC6A4 gene, 5HTT/SLC6A4 gene serotonin transporter gene, NT-3 neurotrophin-3, $P 11$ P11 protein, $P I 3 K$ phosphatidylinositol 3-kinase, $P K A$ protein kinase A, $P K B / A k t$ protein kinase $\mathrm{B}, P K C$ protein kinase C, $P O M C$ proopiomelanocortin, $Q K I$ oligodendrocyte-specific RNA binding protein, SSAT spermidine/spermine $N^{1}$-acetyltransferase $1, T P H 1$ and TPH2 tryptophan hydroxylase 1 and $2, \operatorname{Tr} k B$ tyrosine receptor kinase type B, TrkB-T1 tyrosine receptor kinase type B variant $\mathrm{T} 1$

suicide completion [11]. More promising results were found for TPH2. Studies on single-nucleotide polymorphisms (SNPs or SNIP) and haplotypes of $T P H 2$ have been carried out and a link between TPH2 and suicide has been demonstrated [S42]. In support of these findings, TPH2 mRNA levels in the VPFC of suicide completers were found to be increased and a specific SNIP in the promoter region of TPH2 (rs-10748185) has a significant effect on TPH2 mRNA expression [13]. These data are consistent with previous findings, indicating a greater amount of TPH 2 mRNA in the raphe nuclei and in the DLPFC of suicide victims [S43, S44]. The TPH2 gene at least certainly is a promising subject which deserves further investigation in order to explain better its role in SB.

The SLC6A4 gene, which codes for the 5-HTT and plays a pivotal role in the regulation of 5-HT activity, has a common polymorphism (5-HTTLPR) in a regulatory region which leads to the presence of a short allele (S-allele) and a long allele (Lallele) [S45]. Several studies have shown fewer presynaptic 5HTT sites in suicide victims, especially in the VPFC, regardless of mood disorder diagnoses [11, 12, S32, S33]. The first studies to investigate a possible relation between 5-HTTLPR and 5HTT binding showed no significant association either in the PFC [S33] or in the periphery (platelets) [S46]. Despite these results, the S-allele seems to be associated with impulsive aggression and SB [14, S47]. Moreover, a relation between S-allele and violent suicide attempts [S48] and violent suicide methods [S49] has been highlighted; thus, the presence of at least one S-allele has been proposed as a predictor for SB [11]. A robust association between $\mathrm{S}$-allele and $\mathrm{SB}$ has been shown in two meta-analyses [S50, S51]. 


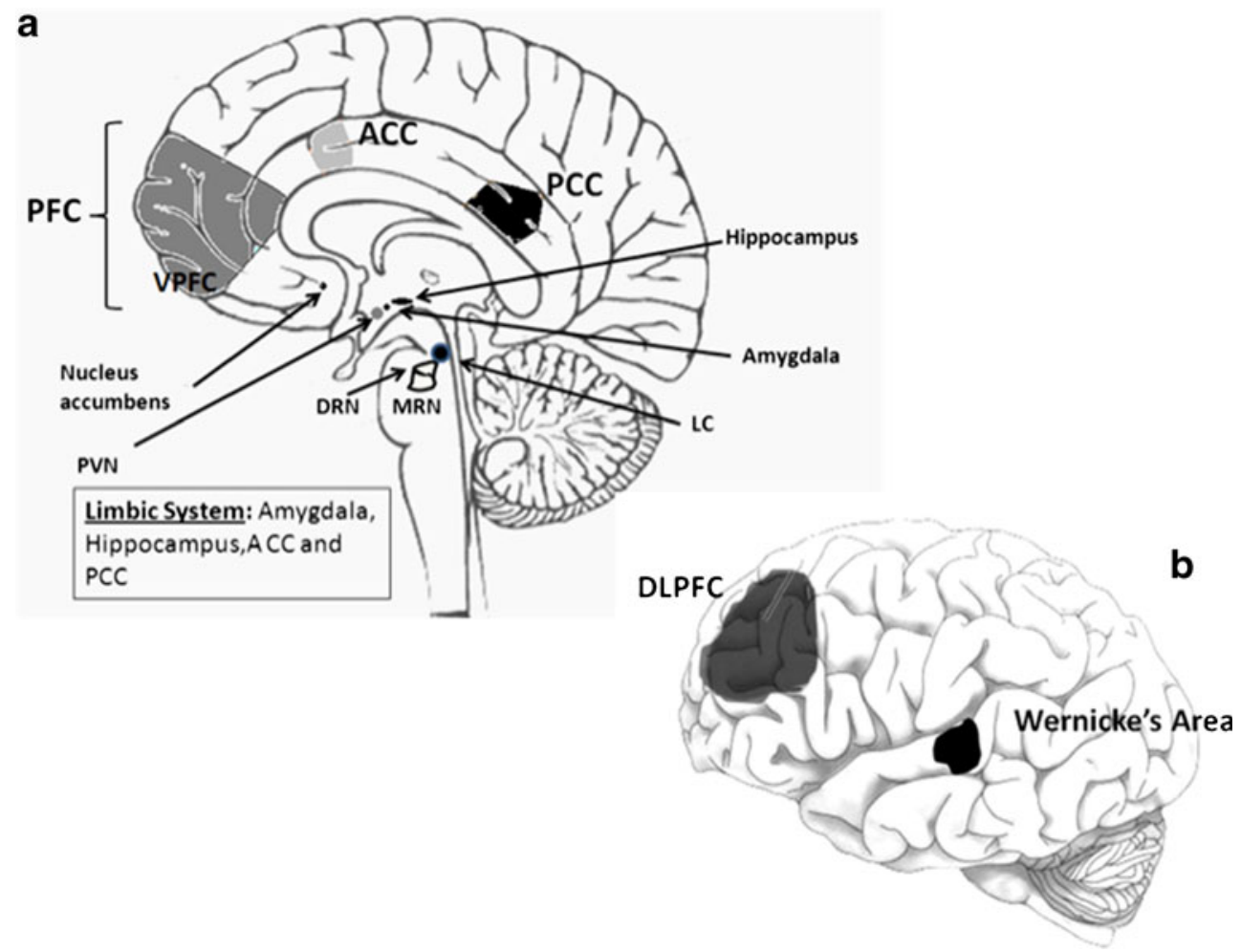

Fig. 1 Main neuroanatomic regions investigated in human brains of suicide completers. a Schematic human brain, sagittal section; b 9chematic human brain, external view. PFC prefrontal cortex [10, 15, 21, 22, S23, S25, S29, S31, S53, S58, S63, S76, S82, S91-S93, S98, S110], $V P F C$ ventral prefrontal cortex, including the orbitofrontal cortex [8, 11-13, S33, S37, S85, S87, S88, S95, S99, S104, S105], $D L P F C$ dorsolateral prefrontal cortex [10, 24, 26, S43, S108, S109], Wernicke's area [20, S89], Hippocampus [18, 19, S58, S82, S85, S91,

Reduced 5-HT neurotransmission in SB may also be recognized through 5-HT receptor (5-HTR) studies. Postsynaptic upregulation of 5-HTR-1A and 5-HTR-2A in the PFC of suicide victims has been demonstrated. This upregulation may be explained as a compensatory response to the low activity of 5-HT neurons [S31], but it is also assumed to be at least partially genetically regulated [S52]. In particular, an increase in 5-HTR-2A binding in Brodmann areas 8 and 9 was found in suicide victims [S31] and there is evidence for the upregulation of 5-HTR-2A in the prefrontal Brodmann areas in suicide brains [S53] as well as in peripheral tissues (platelets of suicide attempters) [S54]. A polymorphism of 5-HTR$2 A$ gene (T102C A) has been associated with suicidal ideation in patients with major depression and it seems that it might confer increased risk of SB [S55], whereas data on the 5-HTR$2 A$ polymorphism are still being debated [S56, S57]. Nevertheless, higher 5-HTR-2A mRNA levels were found in the $\mathrm{PFC}$ and the hippocampus of teenage suicide victims [S58]. Another 5-HTR-2A gene polymorphism (A1438G) has been widely investigated for an association with $\mathrm{SB}$, but no reliable result has emerged, especially for suicide completion [S39, S59]. No association between 5-HTR-1B gene polymorphisms
S94, S96, S98, S110, S111], Amygdale [S122], ACC anterior cingulate cortex [10, 25, S25, S120, S121], Limbic system hippocampus, amygdala, ACC, and posterior cingulated cortex (PCC) [S25], Nucleus accumbens [10], LC locus coeruleus [7, S78], MRN median raphe nucleus [S41, S78], DRN dorsal raphe nucleus [S40, S41, S43, S44], $P V N$ hypothalamic paraventricular nucleus [S73, S74], NC nucleus caudatus [S64]. Some authors have not been specified in this diagram because they examined several brain regions [S26, S27, S79]

and suicide has been detected [S60]. Regarding 5-HTR-2C, alterations in pre-mRNA editing and expression may be found in receptors in the PFC of suicide victims [S58]. Investigation of the variations in genes coding for 5-HTR $1 \mathrm{D} \alpha$ found no differences in allelic or genotypic distributions between suicide completers and controls [S61].

\section{Other neurotransmitters}

Dysregulated expression of GABAergic genes has been found in suicide completers. Altered expression of several GABA receptor subunits was demonstrated in PFC and limbic brain regions. GABA-A receptor-associated protein like 1 (GABARAPL1) showed alterations in gene expression, as well as GABA transporter (SLC6A1) [8, 9, S25, S26, S62, S63].

A study on NMDA receptors, a type of ionotropic glutamate receptor, showed no difference in glutamate receptors between suicide and control subjects. The density of AMPA receptors, another type of ionotropic glutamate receptor, may be increased in the caudate nucleus of suicide subjects [S64]. Glutamatergic genes were found to be altered in mood disorders, but independently of suicide [S62]. 
Adrenergic transmission has been hypothesized to play a role in suicide. The main findings related to the decreased noradrenalin levels in the brainstem and increased $\alpha 2$ adrenergic receptor densities due to a noradrenaline deficit [11]. Abnormalities in $\alpha 2$-adrenergic signaling pathways, possibly associated with factors affecting the $G$ protein cycle, were found in the frontal cortex of depressed suicide victims [S65].

In spite of the dopaminergic theory of mood disorders, there is very little evidence to implicate dopaminergic transmission in suicide and no significant difference in the measurements of dopamine (DA) concentrations in the cortical and subcortical regions in suicide victims versus controls has been found [S66]. The presence of DA D2 receptors might confer a risk of SB in patients with high familiar loading for alcoholism [11]. Anyway, this circuit deserves more investigation, as the significant reduction in DA transport coupled with the observed increase in D2/D3 receptors in the amygdale of subjects with major depressive disorder (MDD) and regional changes in dopaminergic transmission may be involved in mood disorders and suicide [7].

At present, there is no evidence to suggest a change in cholinergic activity in suicide [7]. No significant difference in muscarinic receptor (mAChRs) activity was found in suicide brains [S67].

The relationship of opioids with suicide was first studied in quantitative autoradiography studies. A higher density of $\mu$-opioids receptors was found in the frontal and temporal cortex in younger suicide completers [S68]. This result was confirmed later by the same technique [S69]. Nevertheless, this topic remains to be investigated, as no difference in $\mu$ opioid density or affinity has been found in the PFC and precentral-postcentral gyrus of suicide completers [15].

\section{Hypothalamic-pituitary-adrenal axis}

The HPA axis represents the major biological infrastructure of the human stress system, and its dysfunctions have been investigated in depressed and suicidal patients [2, 7, S58].

Whereas an association between cortisol nonsuppression at the dexamethasone suppression test (DST) and suicide attempt is still controversial [2, S70], cortisol nonsuppression has been strongly associated with suicide completion. It seems to be a useful predictor of suicide completion in depressed subjects [16, S71]. DST nonsuppression emerged as a longterm suicide predictor, while a low level of 5-HIAA in the CSF was associated with short-term suicide risk [S72].

Postmortem studies have shown that hyperactivity of the corticotrophin-releasing hormone $(\mathrm{CRH})$ in the hypothalamic paraventricular nucleus is a prominent feature in depressed subjects and suicide completers [2], as suggested by increased $\mathrm{CRH}$ neuronal counts [S73] and increased CRH mRNA levels
[S74]. Proopiomelanocortin mRNA is also higher in the pituitary corticotropic cells of suicide victims than in controls [S75]. Suicide completers also show reduced CRH binding sites in the PFC [S76] and altered CRH receptor type I and type II ratios in the pituitary gland [S77]. In depressed suicides, augmented CRH immunoreactivity and CRH mRNA levels [S78, S79] as well specific reduction of $\mathrm{CRH}$ receptors $\left(\mathrm{CRH}_{1}\right.$ but not $\left.\mathrm{CRH}_{2}\right)$ have been observed, possibly as a consequence of the elevated CRH levels [S80].

There is increasing evidence that early life environment influences reactivity to stress partly by epigenetic mechanisms that regulate the activity of the genes involved in stress response systems $[2,7,17]$. DNA methylation of the $\mathrm{NR} 3 \mathrm{Cl}$ promoter of the glucocorticoid receptor $(G R)$ exon $1_{\mathrm{F}}$, decreased GR mRNA, as well as mRNA transcripts bearing the GR $1_{\mathrm{F}}$ splice variant were found in the hippocampal tissue of suicide victims with a documented history of childhood abuse, whereas these patterns were not observed in suicide victims without childhood abuse [18]. Consistent with the finding that psychopathology has no effect [18], differences in $1_{\mathrm{F}}$ promoter were not observed in the hippocampus of patients with MDD without a history of childhood abuse who died by causes other than suicide [S81]. The decreased NR3C1 glucocorticoid expression caused by the silencing activity of this methylation results in hyperactivity of the HPA axis, which became unresponsive to the negative feedback exerted by glucocorticoids. GR promoter methylation and differential GR exon $1_{\mathrm{B}}$, $1_{\mathrm{C}}$, and $1_{\mathrm{H}}$ expression have recently been observed in the hippocampus of suicide completers with a history of childhood abuse compared with suicides without a history of abuse and with control subjects [19].

\section{Neurotrophic factors}

Neurotrophic factors, also called neurotrophins, represent a family of extracellular signaling molecules well-known for being implicated in neuronal proliferation, survival, and plasticity. Many investigators have associated them with major depression or suicide and it has been suggested that alterations in their expression partly underlie the changes in plasticity observed in the brains of suicide victims [2, 7]. In this review, the possible role of brain-derived neurotrophic factor (BDNF), tropomyosin-related kinase $\mathrm{B}$ (TrkB), a transmembrane receptor binding to the BDNF, neurotrophin-3 (NT-3), and fibroblast growth factor receptors (FGFR) will be discussed.

Some studies in suicide completers, most of whom were diagnosed with major depression, showed that $B D N F$ and $\operatorname{Trk} B$ were downregulated in different brain regions [S82S84]. Significantly decreased BDNF levels both in the hippocampus and VPFC and decreased NT-3 in the hippocampus, but not in the entorhinal cortex, were observed in suicide victims who did not receive drug treatment [S85]. 
The absence of change in BDNF and NT-3 levels in drugtreated suicide victims may suggest that both neurotrophins are mediators of psychotropic drugs [S85]. Receptors of FGF (FGFR3 and FGFR2) were shown to be downregulated in the PFC and multiple other brain regions of suicide brains [7, S63].

Epigenetic studies in rats exposed to stress showed hypermethylation of several exons in the promoter region of $B D N F$, including exons IV and IX, compared to controls [S86]. After these data had been obtained in animals, a postmortem study on brains from suicide completers found increased $B D N F$ promoter/exon IV methylation in Wernicke's area [20]. Although these results are consistent with some previous BDNF findings from the rat model of early life adversity, a history of childhood abuse was not examined in this study however [2]. A variant of TrkB (TrkB-T1), mostly specific to astroglial cells and with a role in calcium signaling, was downregulated in the orbital frontal cortex of suicide completers in association with methylation at two sites in the promoter, with no alteration in the two main TrkB variants [S87, S88]. This methylation pattern may be specific to the PFC because it was not observed in Wernicke's area or in the cerebellum [2, S89].

Other extracellular and intracellular cells signaling systems

Other extracellular signaling cascades and related intracellular molecules, such as the phosphoinositide and adenylyl cyclase signaling systems, have received attention in mood disorders and suicide [7, 8, S58]. Postmortem studies in depressed patients and suicide completers focused on the possible role of protein kinase C (PKC) [S90-S92], protein kinase A [S93, S94], cyclic AMP-responsive element binding protein [S95S98], protein kinase B (PKB, also called Akt) and glycogen synthase kinase-3 $\beta$ (GSK-3 $\beta$ ) [21, S99], and phosphatidylinositol 3-kinase (PI3K) and phosphatase and tensin homolog (PTEN) [21]. A decrease in Akt and an increase in GSK-3 $\beta$ were found in depressed suicide victims and nonsuicide subjects showing an association with MDD rather than with suicide [S99]. In the same way, in relation to PI3K and PTEN, a decreased PI3K activity and increased protein levels of PTEN were found in depressed subjects, regardless of suicide [21]. A recent paper [22] focused on P11 protein, also called S100A10, which is a regulator of a number of cellular processes, on the basis of its documented association with depression [S100] and posttraumatic stress disorder [S101, S102]. P11 mRNA levels were significantly lower in the peripheral blood mononuclear cells of suicide attempters and in the PFC in suicide completers [22]. Several interactions between these pathways as well as between the molecules and neurotransmitter systems and genes that have been associated with suicide exist, for example, the activation of transcription factors by PKC results in the transcription of $B D N F$
[23]; PKB/akt represents a downstream effector of the 5-HT system [S99], and 5-HT1B receptor function is altered by P11 in depression-like states [S100].

\section{Polyamines}

The function of polyamines in cells is not entirely clear, but it is known that they play a role in cell stress system response and their dysfunctions have been associated with psychopathology both in human and animal studies [7, S103]. In suicide completers with or without major depression, spermidine/spermine $N^{1}$-acetyltransferase 1 (SSAT, also called SAT1 or SAT) expression has been shown to be significantly downregulated in the VPFC [S104, S105] and other brain cortex regions [S27]. Several SNPs in SSAT have been associated with suicide in French Canadians and specific haplotypes have been shown to modify its expression [S104, S106, S107]. DNA methylation at the promoter region of the SSAT gene has been shown to be negatively correlated with its expression in suicide completers [24, S108, S109]. A previous study, in contrast, observed no epigenetic differences between suicide victims and controls [S105]. Possible explanations for these discrepant results may be differences in the psychiatric diagnosis of the subject included, the majority of whom had a major depressive episode [S109] versus a range of axis I disorders [S105] and examination of the DLPFC [S109] versus the VPFC [S105, S109].

\section{Neuropathological data}

Both postmortem genetic and neuropathological studies in mood disorder patients and suicide completers have largely focused on tissues obtained from the PFC, VPFC, including the orbitofrontal cortex, and DLPFC or the limbic area (hippocampus, amygdale, and cingulate cortex) $[9, \mathrm{~S} 110, \mathrm{~S} 11]$ (for a summary of the main neuroanatomic regions investigated in human brains of suicide completers, see Fig. 1).

Alterations of glial cells, especially astrocytes and oligodendrocytes, have been investigated in major depression and suicide [7]. A number of studies in depressed and suicide completer subjects suggested that astrocyte levels were normal or increased in the depressed brain, with the possible exception of younger suicides where a reduction in astrocytes has been observed [S112-S115]. Dysfunction of astrocytes, rather than reduction in cell number, was investigated through microarray analyses for a number of astrocytic-specific genes (i.e., Trkb.T1, FGFR2, FGFR3) [S63, S87, S88] (see the "Neurotrophic factors" section). Given the role of glutamate in mood disorder and the role of astrocytes in its metabolism, astrocytes may be mediators of the glutamate dysfunction detected in major depression and suicide [7, S116]. Hypertrophic astrocytes, with significantly larger cell bodies, as well as 
longer and more ramified processes, were found in the anterior cingulate cortex (ACC) of depressed suicides [25]. At the same time, evidence of the dysfunction of astrocyte connexins 30 and 43 in the DLPFC of suicide completers was provided [26]. This finding is consistent with a gene expression study performed in the locus coeruleus of depressed subjects, most of whom died by suicide [S116].

The hypothesis that oligodendrocyte dysfunctions play a role in depression and suicide was suggested by some reports which found a large number of oligodendroglial-specific genes to be downregulated in the postmortem brain of depressed subjects [S117-S119]. In the ACC, neuronal and glial densities as well as neuronal soma sizes were unaltered in depressed suicides compared to matched controls [S120]. A robust increase in glial cell densities in alcohol-dependent depressed suicides compared with non-alcohol-dependent depressed suicides and controls was found instead [S120]. The same group reported that, in the ACC of depressed suicides, pyramidal neurons displayed altered dendritic branching, with third-order branches significantly reduced in number [S121].

A final aspect of the neuropathologic discussion about the neurobiology of SB concerns neurodevelopmental and neuroplasticity issues [2, 27]. A haplotype of 14-3-3 epsilon, a gene related to neurogenesis, was found to be associated with suicide completion [S122]. Subventricular zone astrocytes are neural stem cells in the adult mammalian brain and it has been suggested that astrocytes play a role in the neurogenesis hypothesis of depression [S123, S124]. Astrocytic-related dysfunctions observed in suicide completers [S63, S87, S88] could influence astroglial growth, signaling, and differentiation [7]. In the same way, the authors who showed alterations in dendritic branching of pyramidal neurons [S121] speculated that this difference may reflect a neurobiological predisposition to depression and suicide given that proximal dendritic branches grow during perinatal development and are generally less plastic at maturity than the distal segments.

\section{Neuroimaging correlates}

In parallel with postmortem genetic and neuropathological findings, both structural and functional neuroimaging studies in SB focused on alterations in the PFC, particularly the VPFC including the orbitofrontal cortex and DLPFC, the ACC, and to a lesser degree, the amygdale [28, S125, S126]. The main correlated neuropsychological traits consist in higher impulsivity, impaired decision-making, a particular attention to specific negative emotional stimuli, lower problem-solving abilities, and reduced verbal fluency [28, 29, S125].

Most recent structural magnetic resonance imaging findings showed a significantly thinner cortex in the VPFC, DLPFC, and ACC in MDD patients at high risk for suicide in contrast to non-high-risk patients [S127]. Specific gray abnormalities in the same regions have been found to discriminate attempter from non-attempter subjects and even to differentiate high-lethality attempts from low-lethality attempts among patients with borderline personality disorder [S128].

Another direction in morphological studies considers the possible role of the white matter [28, S126]. A reduced size of the posterior third of the corpus callosum, with a possible diminished interhemispheric connectivity [S129], and a decreased fractional anisotropy in the left anterior limb of the internal capsule [S130] have been found in patients with SB. When these observations and the glia-related dysfunctions discussed in the "Neuroimaging correlates" section are considered, it may be postulated that a relevant aspect of suicidality neurobiology involves alterations in the glia and, by extension, in the regions from which glial cells are derived and in those regions which contribute fiber tracts [27].

Functional and pharmacological neuroimaging studies have put particular emphasis on the importance of the PFC and the amygdale, as well as their close relationships with the serotoninergic system. Positron emission tomography imaging showed a reduced activation of the medial PFC in major depression patients with high-lethality versus lowlethality suicide attempts [S131]. An increased binding of the 5-HT transporter was found in the ACC of bipolar patients with a history of suicide attempts compared with those without a past history of attempts [S132]. Some studies combining neuroimaging and genetics suggested that the brain regions involved in vulnerability to SB are modulated by genetic polymorphisms previously associated with SB. For instance, the 5HTTLPR variant seems to modulate the activation of the medial PFC and amygdale during both the resting state and aversive stimulation [S133, S134].

\section{Concluding remarks}

The value of investigating the neurobiological issues of suicide consists in the potential for uncovering biomarkers associated with suicide risk. A secondary aspect is the scope for defining some potential biological hallmarks of suicide that could help in determining the circumstances of death.

Concerning neurotransmitters systems, data remain globally contradictory. The most interesting is the 5-HT system, particularly with respect to polymorphisms such as 5-HTTLPR. Despite this, there is no evidence of specificity between a polymorphism and its clinical expression. Among other neurotransmitters, more consistent data are provided only for GABA. The opioid system represents a promising avenue.

To date, the possibility of utilizing results from postmortem studies is mainly limited by the fact that they are not easily generalized in living persons. At a theoretic level, potential indices of suicide risk assessment in peripheral tissues obtained from patients with SB may include the determination of 
epigenetic changes [20, 24, S8, S87, S88, S108, S109]. In particular, epigenetic changes for both HPA axis and BDNF with its receptor TrkB seem to be valid candidates for suicide risk assessment. However, it appears to be difficult to ascribe the observed differences specifically to suicide. Indeed, studies in both animals and humans have indicated that these differences may be better explained by a deleterious environment during childhood. The study conducted by McGowan et al. [18], which shows that suicide subjects who did not suffer childhood abuse do not present any patent change of the HPA axis compared to controls, is an impressive demonstration of this. Because of the important role played by the environment -with not only distal but also proximal effects - more precise and predictive studies on suicide should integrate robust environmental measures, including the longitudinal assessment of developmental factors [2, 7, S135].

Assay of BDNF in the serum [S124] or in peripheral blood lymphocytes and platelets [S136] as well BDNF mRNA assay in peripheral blood mononuclear cells [S136] may represent other theoretical alternatives. The specificity of these indicators, however, is largely limited in the previously mentioned studies because of the significant overlapping with major depression, the more important proxy of suicide risk. This remark may also be generalized to other associations between indices of low 5-HT neurotransmission and SB, which remain unspecific [7]. In this context, long-term prospective studies may be useful. The ideal design of these studies may consist in evaluating two cohorts of patients affected by the same psychiatric disorder, one with SB or suicide completion and the other without. In addition, it has been suggested that other environmental factors such as antidepressant drugs, which are rarely taken into account in these studies, may substantially modify the neurotrophin levels in postmortem brains [S85]. Significant differences in BDNF and NT-3 levels between suicide completers and controls were observed only in those subjects who did not receive drug treatment [S85]. This may prompt some reservations concerning the pertinence of these indices to suicide risk assessment, given that treated subjects will not be detected. P11 protein dosage in peripheral blood mononuclear cells may constitute another alternative [22], but to date, this possibility has been demonstrated in a single study only and needs to be tested in other clinical settings.

Neuropathological data are scarce and not conclusive. An important limitation may consist in the heterogeneity of the clinical patterns included, which may be similar to that discussed for the markers of neuropathological state applied in other psychiatric diseases [S111, S137].

Moreover, the majority of postmortem studies present some methodological limitations, i.e., small sample size or a focus on a single or few brain areas with a relevant influence on the significance of genetic and association studies [S20, S26, S27, S79].
A recent very important direction in the domain of psychiatric genetic studies of suicide is the considerable interest accorded to identifying the underlying dimensions that mediate the relationship between genes and SB, the so-called intermediate phenotypes or endophenotypes [7, S138, S139]. A number of potential candidate biological and clinical endophenotypes for SB have been identified, based largely on association with the phenotype [S139-S141]. Among the impulsive-aggressive traits, disadvantageous decision-making has been suggested as a possible important endophenotype [29, 30, S141-S144]. They represent a very interesting field of investigation in suicide studies, but also raise the problem of exploring these dimensions in dead persons. In this context, psychological autopsies may provide a relevant contribution.

In conclusion, despite the mass of data that has accumulated to date which explores the interaction between the different genetic, environmental, microstructural, and developmental brain factors constituting the complexity of the suicide phenomenon, no formal consensus exists in associating a neurobiological mechanism with suicide. We are still some way from determining a specific biologic abnormality, both in assessing suicide risk and in providing an additional tool to medical experts to correlate the cause of death with suspected suicide.

Acknowledgments This study was supported by the AXA Research Fund, SNF Grant 320030_132853.

Conflict of interest Nader Perroud is on the advisory board for Lundbeck. The other authors declare that they have no conflict of interest.

\section{Glossary of the main genetic terms contained in the text [S30]}

Allele: one of the different forms of a gene that can exist at a single gene locus.

Association mapping: a method used for direct identification of the specific genes (see candidate gene) that control the differences in phenotype among members of a population.

Candidate gene: a gene that, because of its chromosomal position or another property, becomes a candidate for a particular function such as disease risk.

DNA methylation: the addition of methyl groups to DNA residues after replication; it is a mechanism of gene transcription regulation. It represents an important heritable epigenetic mark (see epigenetic).

Endophenotype: quantifiable biologic or psychological variable associated with genetic risk for a disorder; also named intermediate phenotype.

Epigenetic: nongenetic chemical changes in histones or DNA which alter gene function without altering the DNA sequence.

Exome sequencing: sequencing of a set of expressed regions of genes (exons). It is performed only for rare SNPs. 
Gene locus the specific place on a chromosome where a gene is located.

Genome-wide association (GWA): association mapping that uses marker genes loci to scan the entire genome for genes contributing to quantitative variation. It does not require an a priori hypothesis on candidate genes.

Haplotype: the combination of alleles at multiple gene loci on the same segment of homologous chromosomes.

Microarray study: analysis of an array of DNA fragments representing all the genes in a genome. This technique simultaneously examines the expression levels of thousands of gene transcripts.

microRNA (miRNAs): a class of functional RNA that regulates the amount of protein produced by a gene; it represents a mechanism of gene expression regulation (post-transcriptional gene repression).

Phenotype: (1) The form taken by some character (or group of characters) in a specific individual. (2) The detectable outward manifestations of a specific genotype.

Polymorphism: the coexistence of two or more common phenotypes of a character.

Single-nucleotide polymorphisms (SNPs or SNIP): a nucleotide pair difference at a given location in the genomes of two or more individuals. They represent the most prevalent types of polymorphisms. Common SNPs: when the less common allele occurs at a frequency of about $5 \%$ or greater; rare SNPs: when the less common allele occurs at a frequency below $5 \%$.

\section{References}

1. Krug EG, Mercy JA, Dahlberg LL, Zwi AB (2002) The world report on violence and health. Lancet 360:1083-1088

2. Turecki G, Ernst C, Jollant F, Labonté B, Mechawar N (2012) The neurodevelopmental origins of suicidal behavior. Trends Neurosci 35:14-23

3. Hawton K, van Heeringen K (2009) Suicide. Lancet 373:13721381

4. Brezo J, Paris J, Vitaro F, Hébert M, Tremblay RE, Turecki G (2008) Predicting suicide attempts in young adults with histories of childhood abuse. Br J Psychiatry 193:134-139

5. Qin P, Agerbo E, Bo Mortensen PB (2003) Suicide risk in relation to socioeconomic, demographic, psychiatric, and familial factors: a national register-based study of all suicides in Denmark, 19811997. Am J Psychiatry 160:765-772

6. Mann JJ (2003) Neurobiology of suicidal behavior. Nat Rev Neurosci 4:819-828

7. Ernst C, Mechawar N, Turecki G (2009) Suicide neurobiology. Prog Neurobiol 89:315-333

8. Klempan TA, Sequeira A, Canetti L, Lalovic A, Ernst C, ffrenchMullen J, Turecki G (2009) Altered expression of genes involved in ATP biosynthesis and GABAergic neurotransmission in the ventral prefrontal cortex of suicides with and without major depression. Mol Psychiatry 14:175-189

9. Fiori LM, Turecki G (2010) Gene expression profiling of suicide completers. Eur Psychiatry 25:287-290
10. Sequeira A, Morgan L, Walsh DM, Cartagena PM, Choudary P, Li J, Schatzberg AF, Watson SJ, Akil H, Myers RM, Jones EG, Bunney WE, Vaeter MP (2012) Gene expression changes in the prefrontal cortex, anterior cingulate cortex and nucleus accumbens of mood disorders subjects that committed suicide. PLoS One 7:e35367

11. Bondy B, Buettner A, Zill P (2006) Genetics of suicide. Mol Psychiatry 11:336-351

12. Leboyer M, Slama F, Siever BF (2005) Suicidal disorders: a nosological entity per se? Am J Med Genet C Semin Med Genet $133 \mathrm{C}: 3-7$

13. Perroud N, Neidhart E, Petit B, Vessaz M, Laforge T, Relecom C, La Harpe R, Malafosse A, Guipponi M (2010) Simultaneous analysis of serotonin transporter, tryptophan hydroxylase 1 and 2 gene expression in the ventral prefrontal cortex of suicide victims. Am J Med Genet Part B 153B:909-918

14. Bondy B, Erfurth A, de Jonge S, Krüger M, Meyer H (2000) Possible association of the short allele of the serotonin transporter promoter gene polymorphism (5-HTTLPR) with violent suicide. Mol Psychiatry 5:193-195

15. Zalsman G, Molcho A, Huang Y, Dwork A, Li S, Mann JJ (2005) Postmortem mu-opioid receptor binding in suicide victims and controls. J Neural Transm 112:949-954

16. Coryell W, Schlesser M (2001) The dexamethasone suppression test and suicide prediction. Am J Psychiatry 158:748-753

17. Perroud N, Paoloni-Giacobino A, Prada P, Olié E, Salzmann A, Nicastro R, Guillaume S, Mouthon D, Stouder C, Dieben K, Huguelet P, Courtet P, Malafosse A (2011) Increased methylation of glucocorticoid receptor gene (NR3C1) in adults with a history of childhood maltreatment: a link with the severity and type of trauma. Transl Psychiatry. doi:10.1038/tp.2011.60

18. McGowan PO, Sasaki A, D’Alessio A, Dymov S, Labonté B, Szyf M, Turecki G, Meaney MJ (2009) Epigenetic regulation of the glucocorticoid receptor in human brain associates with childhood abuse. Nat Neurosci 12:342-348

19. Labonté B, Yerko V, Gross J, Mechawar N, Meaney MJ, Szyf M, Turecki G (2012) Differential glucocorticoid receptor exon $1_{B}, 1_{C}$, and $1_{\mathrm{H}}$ expression and methylation in suicide completers with a history of childhood abuse. Biol Psychiatry. doi:10.1016/ j.biopsych.2012.01.034

20. Keller S, Sarchiapone M, Zarrilli F, Videtic A, Ferraro A, Carli V, Sacchetti S, Lembo F, Angiolillo A, Jovanovic N, Pisanti F, Tomaiuolo R, Monticelli A, Balazic J, Roy A, Marusic A, Cocozza S, Fusco A, Bruni CB, Castaldo G, Chiariotti L (2010) Increased BDNF promoter methylation in the Wernicke area of suicide subjects. Arch Gen Psychiatry 67:258-267

21. Karege F, Perroud N, Burkhardt S, Fernandez R, Ballmann E, La Harpe R, Malafosse A (2011) Alterations in phosphatidylinositol 3-kinase activity and PTEN phosphatase in the prefrontal cortex of depressed suicide victims. Neuropsychobiology 63:224-231

22. Zhang L, Su TP, Choi K, Maree W, Li CT, Chung MY, Chen YS, Bai YM, Chou YH, Barker JL, Barrett JE, Li XX, Li H, Benedek DM, Ursano R (2011) P11 (S100A10) as a potential biomarker of psychiatric patients at risk of suicide. J Psychiatr Res 45:435-441

23. Pandey GN, Dwivedi Y (2010) What can post-mortem studies tell us about the pathoetiology of suicide? Future Neurol 5:701-720

24. Fiori LM, Turecki G (2010) Genetic and epigenetic influences on expression of spermine synthase and spermine oxidase in suicide completers. Int J Neuropsychopharmacol 13:725-736

25. Torres-Platas SG, Hercher C, Davoli MA, Maussion G, Labonté B, Turecki G, Mechawar N (2011) Astrocytic hypertrophy in anterior cingulated white matter of depressed suicides. Neuropsychopharmachology 36:2650-2658

26. Ernst C, Nagy C, Kim S, Yang JP, Deng X, Hellstrom IC, Choi KH, Gershenfeld H, Meaney MJ, Turecki G (2011) Dysfunction of astrocyte connexins 30 and 43 in dorsal lateral prefrontal cortex of suicide completers. Biol Psychiatry 70:312-319 
27. Underwood MD, Arango V (2011) Evidence for neurodegeneration and neuroplasticity as part of the neurobiology of suicide. Biol Psychiatry 70:306-307

28. Jollant F, Lawrence NL, Olié E, Guillaume S, Courtet P (2011) The suicidal mind and brain: a review of neuropsychological and neuroimaging studies. World J Biol Psychiatry 12:319-339
29. Courtet P, Gottesman II, Jollant F, Gould TD (2011) The neuroscience of suicidal behaviors: what can we expect from endophenotype strategies? Transl Psychiatry 1(5):e7. doi:10.1038/tp.2011.6

30. Turecki G (2005) Dissecting the suicide phenotype: the role of impulsive-aggressive behaviors. J Psychiatry Neurosci 30:398408 